Check for updates

Cite this: RSC Adv., 2019, 9, 145

Received 27th August 2018

Accepted 12th December 2018

DOI: $10.1039 / c 8 r a 07157 h$

rsc.li/rsc-advances

\section{Versatile cross-linked fatty acid-based polycarbonate networks obtained by thiol-ene coupling reaction}

\author{
Pierre-Luc Durand, ${ }^{a}$ Guillaume Chollet, ${ }^{b}$ Etienne Grau ${ }^{a}$ and Henri Cramail (iD *a
}

\section{Introduction}

Over the last few decades, the interest for polymers stemming from renewable resources as substitutes to oil-based polymers has been continuously growing both in academia and industry. ${ }^{1}$ This new trend is also a way to develop synthetic strategies following the green chemistry principles in view of a sustainable development.

Among the available bio-resources, vegetable oils appear as a very versatile platform for the design of polymer building blocks. ${ }^{2,3}$ Castor oil is one of the main renewable feed-stocks allowing the synthesis of bio-based polymeric materials, including, polyesters, polyamides, polyurethanes and many others. $^{4}$

Recently, methyl 10-undecenoate, a commercially available castor oil derivative, was shown to be a suitable starting building-block to design original functional aliphatic polycarbonate (Fig. 1). ${ }^{5}$

In order to enhance the thermo-mechanical properties of these aliphatic polycarbonates, the present work is dedicated to the synthesis of versatile networks from these linear polycarbonate precursors.

Such polycarbonate networks exhibiting elastomeric properties are desirable thanks to a large number of applications in the biomedical area, especially in the emerging fields of softtissue engineering or drug delivery. ${ }^{6-11}$

${ }^{a}$ Laboratoire de Chimie des Polymères Organiques, UMR 5629, CNRS, Universitè de Bordeaux, Bordeaux INP/ENSCBP, 16 Avenue Pey Berland, 33600, Pessac, France. E-mail: cramail@enscbp.fr

${ }^{b}$ ITERG, F-33600, Pessac, France
Due to the presence of one dangling unsaturation per monomer units, thiol-ene coupling reaction appears to be a suitable method for the preparation of cross-linked networks.

Indeed, radical thiol-ene addition has been extensively employed for the cross-linking of functional polymers. For instance, Stevens et al. have exploited the thiol-ene reaction for the formation of nanosponges, through the cross-linking of copolymers. $^{12}$ Truong et $a .^{13}$ and Stevens et al. ${ }^{14}$ developed hydrogels with tunable properties including water uptake content, mechanical strength and degradation time by crosslinking poly(5-methyl-5-allyloxycarbonyl-trimethylenecarbonate) (PMAC) with a range of PEG-thiols. Finally, Barker et al. ${ }^{15}$ have used photo-initiated radical thiol-ene chemistry to produce $3 \mathrm{D}$ printed structures based on polycarbonates via microstereolithography, as an alternative to potentially toxic acrylatebased printing systems.

The aim of this work is thus to investigate the influence of several parameters on the fatty acid-based polycarbonate network properties. First, different cross-linkers were used and investigations were performed to study their influence on the network properties. The influence of the cross-linker/olefin unit ratio on the network properties was also analyzed.

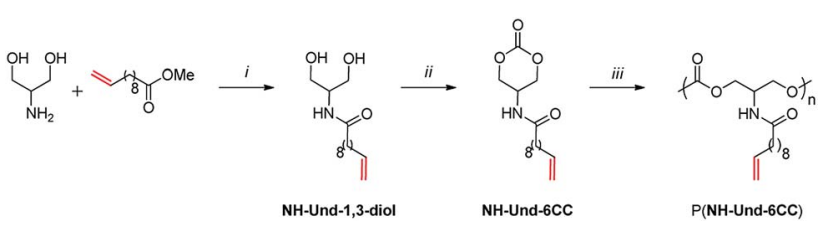

Fig. 1 Synthesis of lipidic polycarbonate from methyl undecenoate and 2-amino-1,3-propanediol. 


\section{Experimental section}

Materials

All products and solvents (reagent grade) were used as received except otherwise mentioned. The solvents were of reagent grade quality and were purified wherever necessary according to the methods reported in the literature. The polycarbonate, $\mathbf{P}(\mathbf{N H}-$ Und-6CC) has been synthesized as previously described. ${ }^{5}$

\section{General considerations}

Polymer molar masses were determined by size exclusion chromatography (SEC) using tetrahydrofuran (THF with $250 \mathrm{ppm}$ of BHT as inhibitor) as the eluent. Measurements in THF were performed on a PL GPC 50 from Agilent equipped with RI and UV detector. The separation was achieved on three Tosoh TSK HXL gel columns $(300 \times 7.5 \mathrm{~mm})$ G4000, G3000 and G2000 with an exclusion limits from 200 to $400000 \mathrm{Da}$, at flow rate of $1 \mathrm{~mL} \mathrm{~min}^{-1}$. The injected volume was $20 \mu \mathrm{L}$. Columns' temperature was held at $40{ }^{\circ} \mathrm{C}$.

The Raman measurements were performed using a JobinYvon Horiba XploRA confocal spectrometer with a resolution of $4.3 \mathrm{~cm}^{-1}$ in the spectral range $900-3100 \mathrm{~cm}^{-1}$. The laser source is a laser diode giving access to two laser wavelengths with a maximum power of $45 \mathrm{~mW}$. Our work was carried out using an incident wavelength of $\lambda=785 \mathrm{~nm}$ and the laser beam power was fixed at $100 \%$ of the maximum power. To improve the signal-tonoise ratio, each spectrum was the result of 2 accumulated spectra with an acquisition time of 30 seconds for each spectrum.

\section{General procedure for the cross-linking process through thiol-ene chemistry}

P(NH-Und-6CC) (1.0 g, $3.53 \mathrm{mmol}, M_{\mathrm{n}}=5700 \mathrm{~g} \mathrm{~mol}^{-1}, M_{\mathrm{w}} / M_{\mathrm{n}}=$ 1.07), 1,6-hexanedithiol ( $264.5 \mathrm{mg}, 1.76 \mathrm{mmol}, 0.5$ equiv.) and a radical initiator (Irgacure 2959, $7.9 \mathrm{mg}, 0.0035 \mathrm{mmol}, 0.01$ equiv.) were dissolved in $1 \mathrm{~mL}$ of DCM and transferred to a Teflon mold. The solvent was gently evaporated overnight and residual DCM was removed under reduced pressure during $4 \mathrm{~h}$. The mixture was then irradiated at $365 \mathrm{~nm}$ during 4 hours. The obtained cross-linked film was flexible and transparent.

To determine equilibrium swelling ratios and gel contents, strip-shaped specimens $(0.6 \mathrm{~mm}$ thick, $0.5 \mathrm{~mm}$ width, $3 \mathrm{~mm}$ length) were placed in $10 \mathrm{~mL}$ of DCM for 24 hours. We assume that this procedure ensured complete removal of the sol fraction. Then the swollen gels were dried to constant weight at room temperature in vacuum and weighted.

The gel fraction $\left(F_{\mathrm{g}}\right)$ was calculated according to eqn (1) where $m_{\mathrm{d}}$ is the mass of dried (extracted) samples and $m_{0}$ is the mass of the specimens before swelling.

$$
F_{\mathrm{g}}(\%)=\frac{m_{\mathrm{d}}}{m_{0}} \times 100
$$

\section{Thermal and mechanical properties}

Differential scanning calorimetry (DSC) measurements of polymer samples ( $\approx 10 \mathrm{mg}$ ) were performed using a Q 100-RCS apparatus from TA Instruments over temperature range from 0 to $230{ }^{\circ} \mathrm{C}$, in a heating cooling mode of $10{ }^{\circ} \mathrm{C} \mathrm{min}^{-1}$. The analyses were carried out in a nitrogen atmosphere with aluminum pans.

Dynamic mechanical analyses (DMA) were performed on RSA 3 (TA instrument). The sample temperature was modulated from $-50{ }^{\circ} \mathrm{C}$ to $80{ }^{\circ} \mathrm{C}$, depending on the sample at a heating rate of $4{ }^{\circ} \mathrm{C} \mathrm{min}{ }^{-1}$. The measurements were performed in a tension mode at a frequency of $1 \mathrm{~Hz}$, an initial static force of $0.1 \mathrm{~N}$ and a strain sweep of $0.04 \%$.

Tensile tests were performed on a MTS Qtest 25 Elite controller (France) at $20^{\circ} \mathrm{C}$. The initial grip separation was set at $20 \mathrm{~mm}$ and the crosshead speed at $50 \mathrm{~mm} \mathrm{~min}^{-1}$. The results were obtained from at least 3 replicates for each sample.

\section{Results and discussion}

We previously reported the synthesis of a bio-based polycarbonate containing dangling unsaturations. ${ }^{5}$ Since the pendent olefin groups are reasonably distributed throughout the polymer structure, we aimed at examining the photoinduced cross-linking of these polycarbonate precursors via thiol-ene chemistry, to prepare bio-based polycarbonate materials with improved thermal and mechanical properties. Indeed, such synthetic methodology was already reported for the functionalization of linear polymers but also as a mean to form cross-linked materials. ${ }^{\mathbf{1 6 - 2 0}}$ With this objective, the linear lipidic polycarbonate, illustrated in Fig. 2, was reacted in DCM with several dithiols in the presence of Irgacure 2959 used as radical initiator in catalytic amounts $(1 \mathrm{~mol} \%)$, to yield transparent flexible materials (Fig. 3).

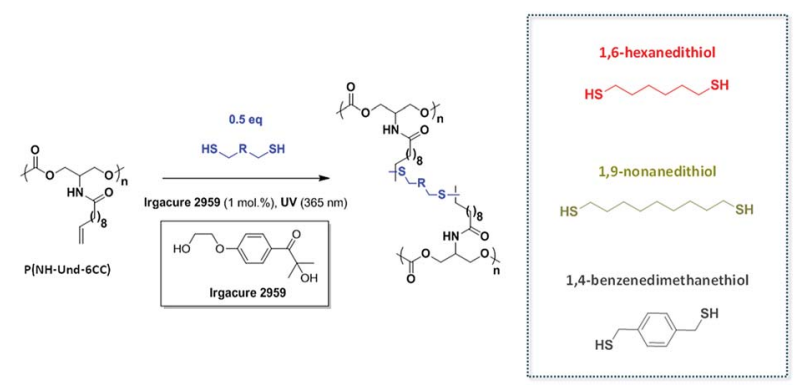

Fig. 2 Cross-linking of $\mathrm{P}(\mathrm{NH}-\mathrm{Und}-6 \mathrm{CC})$ via thiol-ene reaction with different cross-linkers.

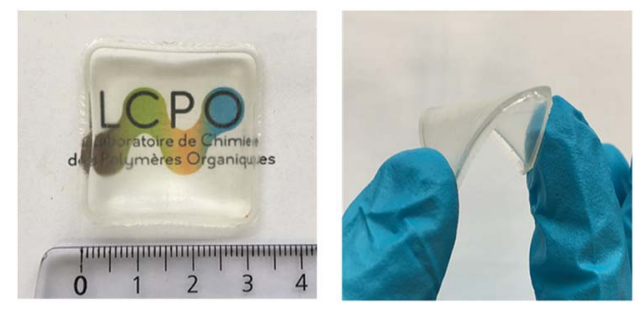

Fig. 3 Bio-based polycarbonate network (Table 1, entry 1). 
The aim of this study was to investigate both the influence of the type of cross-linker used and the cross-linker/olefin ratio on the polycarbonate network properties.

First, different cross-linkers were compared for a constant ratio dithiol/olefin ratio of 0.5 in order to get the highest crosslinking density (Fig. 2). P(NH-Und-6CC) was selected as starting polycarbonate, Irgacure 2959 as photoinitiator and 1,6-hexanedithiol (HDT), 1,9-nonanedithiol (NDT) and 1,4-benzenedimethanethiol (BDT) were used as cross-linkers. The use of 1,9nonanedithiol should induce more space between the reactive groups and 1,4-benzenedimethanethiol should bring some rigidity between cross-linking points.

The mechanical properties have been evaluated by DMA and tensile tests. Results are summarized in Table 1.

All the materials display high gel content $(\geq 89 \%)$ and rather low smell of dithiol testifying an almost complete cross-linking reaction. However, surprisingly, the network obtained with 1,9nonanedithiol (NDT) exhibits a higher $T_{\mathrm{g}}\left(37.7^{\circ} \mathrm{C}\right)$ and better mechanical properties (Young modulus of $240 \mathrm{MPa}$ ) than the network formed with 1,6-hexanedithiol (HDT) $\left(T_{\mathrm{g}}=15.1{ }^{\circ} \mathrm{C}\right.$ and Young modulus of $50 \mathrm{MPa}$ ) as seen from DMA curves (Fig. 4a) and stress-strain traces (Fig. 4b). Indeed, above the glass transition temperature, the storage modulus was much higher with NDT than HDT (increased from 4.5 to 8.9 MPa). On the contrary, when the rigid 1,4-benzenedimethanethiol (BDT) was used instead of HDT, a decrease of the network $T_{\mathrm{g}}$ (from 15.1 to $8.8{ }^{\circ} \mathrm{C}$ ) and mechanical properties (storage modulus decreases from 4.5 to 0.9 $\mathrm{MPa}$ ) was observed. The rigidity of the aromatic ring and the relative proximity of the reactive groups in 1,4-benzenedimethanethiol may hinder the access of the thiols to pendent olefins. In such a case, intramolecular coupling could occur resulting in a less cross-linked network. In contrast, the nine-carbon distance between the two thiol groups in 1,9-nonanedithiol makes carboncarbon double bond more accessible. The so-formed network is thus fully cross-linked and displays higher mechanical properties, as confirmed by DMA tests. Indeed, the rubbery modulus plateau, which is proportional to the crosslinking density, is smaller for HDT than for NDT.

These first experiments show that thermo-mechanical properties of the polycarbonate materials can be adjusted with respect to the type of cross-linker used (Fig. 5).

In the following, 1,6-hexanedithiol (HDT) was selected as a cross-linker to investigate the effect of the cross-linker concentration on the network feature. In theory, 0.5 equivalent of dithiol compared to the unsaturation should be added to entirely consume the total amount of unsaturations. However,
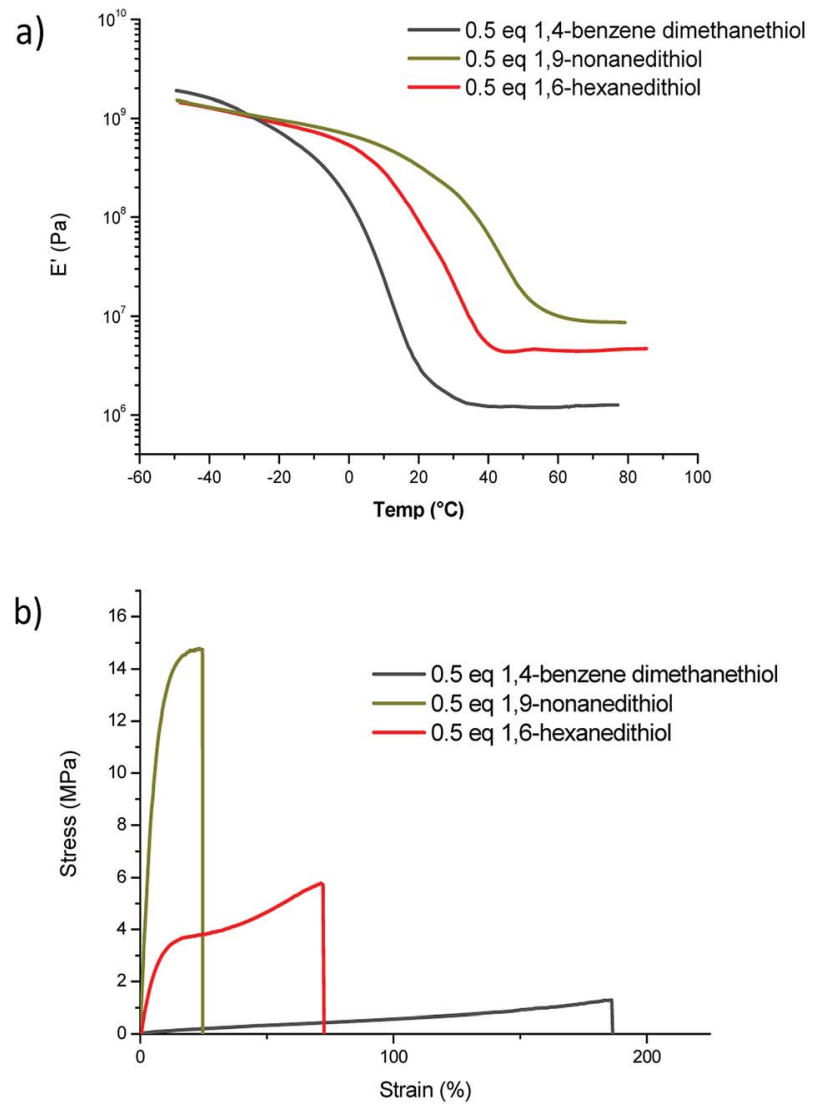

Fig. 4 (a) DMA curves and (b) stress-strain traces of polycarbonate networks obtained with different cross-linkers.

in order to tune the 3D-polycarbonate properties, different cross-linker ratios have been investigated (Table 2).

The effect of the cross-linker concentration on gel contents and swelling ratios can be clearly seen. As expected, the gel contents and swelling ratios of the polycarbonate networks vary strongly with the cross-linker content. Indeed, the gel fraction of the network increases from $34 \%$ to $89 \%$ with an increase of HDT from 0.1 equiv. to 0.5 eq. Simultaneously, the swelling ratio in dichloromethane of the polycarbonate network decreased from $283 \%$ to $97 \%$. It is important to note that an excess of cross-linker ( 0.8 equiv.) greatly increases the swelling ratio without decreasing the gel content meaning that the cross-linking density is lower.

As expected, the $T_{\mathrm{g}}$ increases when the dithiol/olefin ratio goes from 0.1 to 0.5 . However, an increase to 0.8 equivalent of dithiol leads to lower $T_{\mathrm{g}}$ and lower storage modulus at the

Table 1 Mechanical properties of fatty acid-based polycarbonate networks cross-linked with different dithiols

\begin{tabular}{|c|c|c|c|c|c|c|}
\hline Cross-linker & $\begin{array}{l}\text { Gel content } \\
(\%)\end{array}$ & $T_{\mathrm{g}}^{a}\left({ }^{\circ} \mathrm{C}\right)$ & $E^{\prime b}(\mathrm{MPa})$ & Young modulus ${ }^{c}(\mathrm{MPa})$ & Elongation at break ${ }^{c}(\%)$ & Max stress ${ }^{c}(\mathrm{MPa})$ \\
\hline HDT & 89 & 15.1 & 4.5 & $50 \pm 4$ & $76 \pm 5$ & $6.1 \pm 0.8$ \\
\hline NDT & 98 & 37.7 & 8.9 & $240 \pm 20$ & $25 \pm 6$ & $29 \pm 3$ \\
\hline BDT & 99 & 8.8 & 0.9 & $1.3 \pm 0.1$ & $190 \pm 5$ & $1.3 \pm 0.1$ \\
\hline
\end{tabular}

${ }^{a}$ Determined by DSC at $10{ }^{\circ} \mathrm{C} \mathrm{min}{ }^{-1}$ from the second cycle. ${ }^{b}$ Evaluated with DMA analysis. ${ }^{c}$ Calculated from tensile tests. 


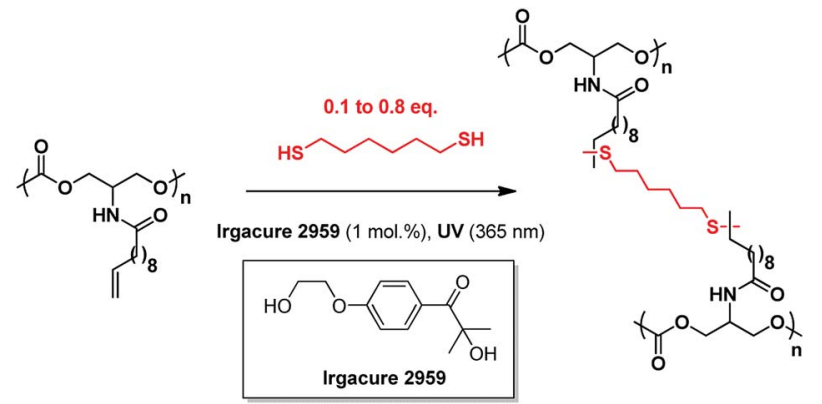

Fig. 5 Cross-linking of $\mathrm{P}(\mathrm{NH}-$ Und-6CC) with different equivalents of 1,6-hexanedithiol.

rubbery plateau, confirming that an excess of dithiol tends to decrease the cross-linking density of the network. It is speculated that pendent thiol groups which could not react with the unsaturations are probably present within the network (Fig. 6). This hypothesis is reinforced by the strong smell of the polycarbonate material even after several washes.

In order to confirm the presence of pendent thiol groups, Raman spectroscopy was performed. The Raman spectrum (Fig. 7) exhibits the characteristic peak of thiol groups (around $2600 \mathrm{~cm}^{-1}$ ) even after several washings with phosphatebuffered saline (PBS), confirming the presence of such dangling functions grafted to the polycarbonate network.

The thermo-mechanical behaviour of the networks was determined by DMA, under tension mode. The storage modulus as a function of temperature for each polycarbonate network is shown in Fig. 9. These traces are indicative of amorphous crosslinked networks. At low temperatures, a glassy modulus plateau at $1 \mathrm{GPa}$ is obtained. The glass transition region is recognized by the drastic decrease in modulus with increasing temperature. Beyond the glass transition region, the rubbery modulus plateau is nearly constant and, in accordance with the rubber elasticity theory, it is proportional to the cross-linking density of the network. We can clearly note, that the storage modulus at $50{ }^{\circ} \mathrm{C}$ decreases significantly from $4.5 \mathrm{MPa}$ to $0.1 \mathrm{MPa}$ by decreasing the number of equivalents of the cross-linker (from 0.5 to 0.1 ).

The difference between elastic modulus values is due to the different cross-link densities $(\nu)$ of networks defined as the number of moles of elastically effective network chains per cubic centimeter of sample, which can be calculated with the

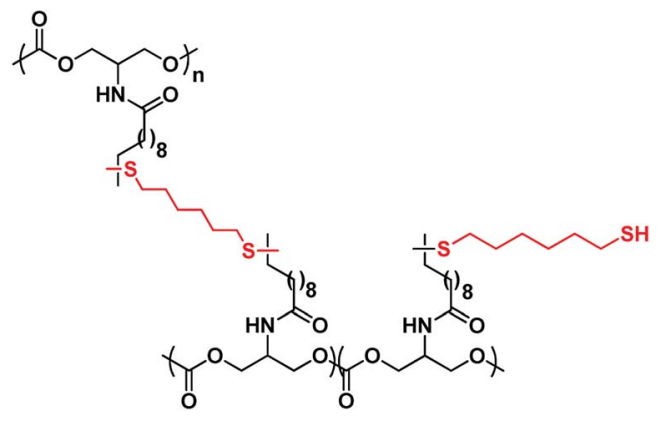

Fig. 6 Structure of the polycarbonate network when 0.8 equiv. of dithiol is added with 1 equiv. of olefin.

following eqn (2) with $R=8314 \mathrm{~J} \mathrm{~mol}^{-1} \mathrm{~K}^{-1}, T_{\alpha+30}=328.15 \mathrm{~K}$ and $\phi=1$.

$$
\nu=\frac{E^{\prime}\left(T_{\alpha+30}\right)}{\phi R T_{\alpha+30}}
$$

Fig. 8 exhibits the effect of cross-linker ratio (from 0.1 to 0.5 ) on the intensity of $\tan \delta$. Interestingly, the value of the alpha transition $\left(T_{\alpha}\right)$ is almost independent of the cross-linking density (around $25{ }^{\circ} \mathrm{C}$ ). However, the intensity of tan delta peak varied significantly from 0.44 to 1.41 for 0.5 and 0.1 equivalent of dithiol, respectively. As the intensity reflects the extent of mobility of polymer chain at this temperature, the fact that $\tan \delta$ intensity increases when the cross-linking decreases is coherent.

In addition, an excess of dithiol ( 0.8 equiv.) results in lowering the storage modulus at the rubbery plateau. This result confirms that an excess of cross-linker decreases the crosslinking density.

Nevertheless, the elasticity properties of the material can be modulated by tuning the cross-linker content.

The tensile properties of the polycarbonate network films are also given in Table 2 while representative stress-strain curves are shown in Fig. 10.

It can be seen from these data that cross-linked polycarbonates exhibit different stress and strain at break values depending on the cross-linking density. Polycarbonate films containing 0.5 equivalent of cross-linker have stress at break values 7 times higher than the ones prepared with 0.1 equivalent of cross-linker (5.7 MPa and 0.8 MPa respectively). Strain

Table 2 Thermo-mechanical properties of cross-linked $\mathrm{P}(\mathrm{NH}-$ Und-6CC) with different dithiol/olefin ratios

\begin{tabular}{|c|c|c|c|c|c|c|c|}
\hline $\begin{array}{l}\text { Dithiol/olefin } \\
\text { ratio }\end{array}$ & $T_{\mathrm{g}}^{a}\left({ }^{\circ} \mathrm{C}\right)$ & $E^{\prime b}(\mathrm{MPa})$ & Young modulus ${ }^{c}(\mathrm{MPa})$ & Elongation at break $k^{c}(\%)$ & Max stress ${ }^{c}(\mathrm{MPa})$ & $\begin{array}{l}\text { Gel content } \\
(\%)\end{array}$ & $\begin{array}{l}\text { Swelling ratio } \\
(\%)\end{array}$ \\
\hline 0.8 & 7.4 & 1.0 & $1.4 \pm 0.5$ & $121 \pm 10$ & $1.2 \pm 0.5$ & 83 & 214 \\
\hline 0.5 & 15.1 & 4.5 & $50 \pm 4$ & $76 \pm 5$ & $6.1 \pm 0.8$ & 89 & 97 \\
\hline 0.3 & 14.7 & 4.2 & $31 \pm 5$ & $64 \pm 1$ & $5.8 \pm 0.5$ & 86 & 102 \\
\hline 0.2 & 8.6 & 2.0 & $2.4 \pm 0.2$ & $134 \pm 12$ & $2.2 \pm 0.2$ & 71 & 180 \\
\hline 0.1 & 5.3 & 0.1 & $0.8 \pm 0.2$ & $175 \pm 20$ & $0.8 \pm 0.1$ & 34 & 283 \\
\hline
\end{tabular}

${ }^{a}$ Determined by DSC at $10{ }^{\circ} \mathrm{C} \min ^{-1}$ from the second cycle. ${ }^{b}$ Evaluated with DMA analysis. ${ }^{c}$ Calculated from tensile tests. 


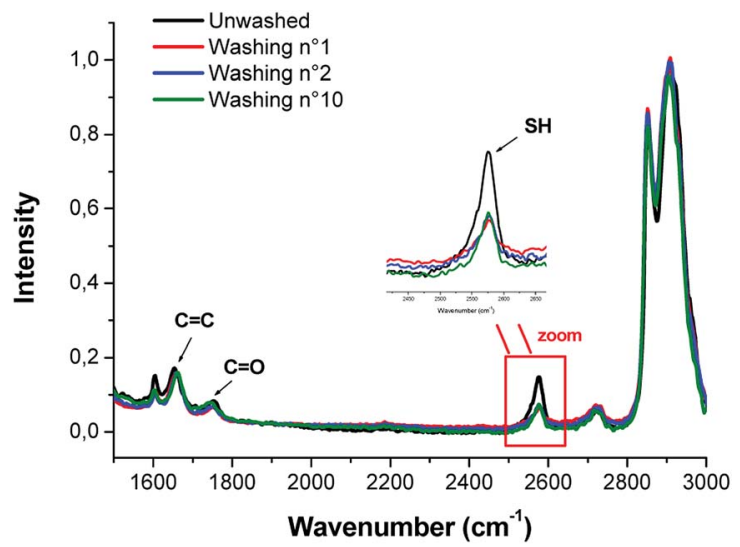

Fig. 7 Titration of the dangling thiol groups in the polycarbonate network by Raman spectroscopy.

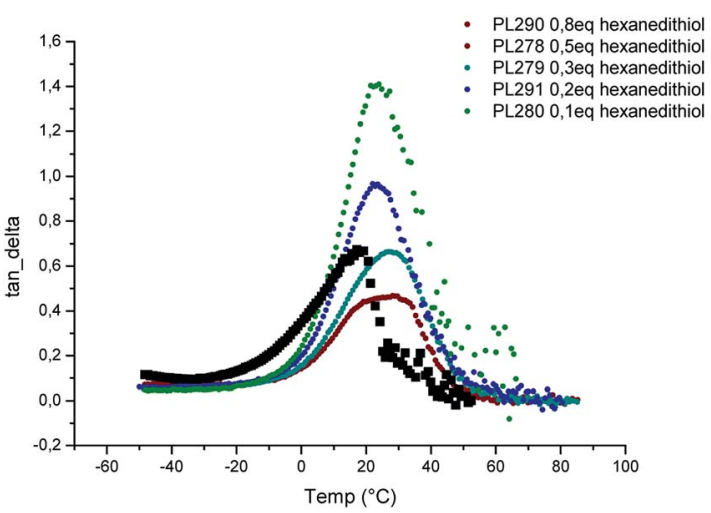

Fig. 8 Effect of cross-linker to olefin ratio on tan delta.

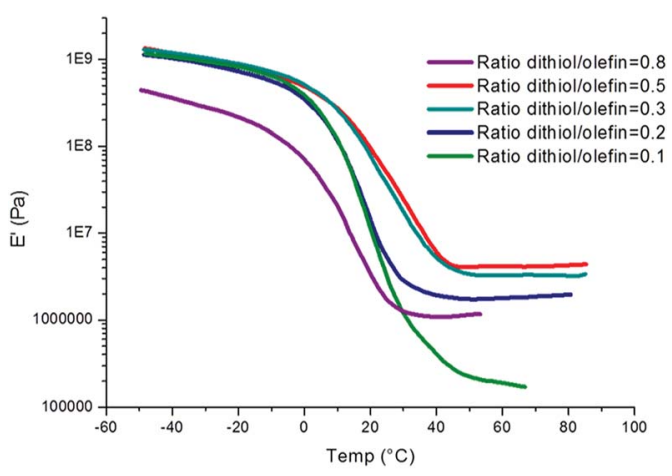

Fig. 9 Storage modulus as a function of HDT/olefin ratio.

at break values can be also modulated depending of the crosslinker content. The range of values goes from around $70 \%$ (with 0.5 equiv. of dithiol) to almost $200 \%$ (with 0.1 equiv. of dithiol). As expected, the excess of cross-linker decreases the mechanical properties of the so-formed polycarbonate network.

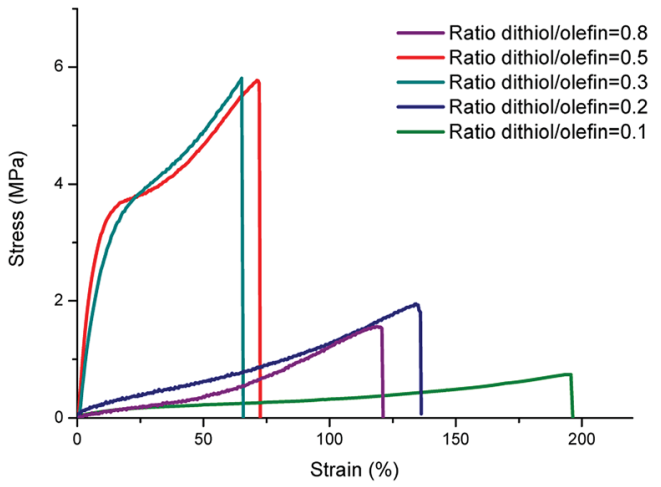

Fig. 10 Representative stress-strain curves of polycarbonate networks with different HDT/olefin ratios.

\section{Conclusion}

In conclusion, the thiol-ene cross-linking method affords the preparation of bio-based aliphatic polycarbonate networks presenting a wide range of mechanical properties depending on the cross-linker nature and content. $T_{\mathrm{g}}$ between $5{ }^{\circ} \mathrm{C}$ to $38{ }^{\circ} \mathrm{C}$ with elongation at break up to $190 \%$ have been obtained. In addition, an excess of dithiol compared to olefin decreases the cross-linking density of the network with no loss in the gel fraction. Nevertheless, the so-formed network possesses pendant thiol groups which can be useful for further chemical modification.

\section{Conflicts of interest}

There are no conflicts to declare.

\section{Acknowledgements}

This work was performed, in partnership with the SAS PIVERT, within the frame of the French Institute for the Energy Transition (Institut pour la Transition Energétique - ITE) P.I.V.E.R.T. (http://www.institut-pivert.com) selected as an Investment for the Future ("Investissements d'Avenir"). This work was supported, as part of the Investments for the Future, by the French Government under the Reference ANR-001-01. The authors thank Equipex Xyloforest ANR-10-EQPX-16. The financial support from the CPER CAMPUSB project funded by the French state and the Région Nouvelle Aquitaine is gratefully acknowledged.

\section{References}

1 A. Llevot, P. K. Dannecker, M. von Czapiewski, L. C. Over, Z. Söyler and M. A. R. Meier, Chem.-Eur. J., 2016, 22(33), 11510-11521.

2 L. Maisonneuve, T. Lebarbé, E. Grau and H. Cramail, Polym. Chem., 2013, 4(22), 5472.

3 Y. Xia and R. C. Larock, Green Chem., 2010, 12(11), 18931909. 
4 H. Mutlu and M. A. R. Meier, Eur. J. Lipid Sci. Technol., 2010, 112(1), 10-30.

5 P. L. Durand, A. Brège, G. Chollet, E. Grau and H. Cramail, ACS Macro Lett., 2018, 7(2), 250-254.

6 M. Martina and D. W. Hutmacher, Polym. Int., 2007, 56, 145157.

7 B. Amsden, Soft Matter, 2007, 3(11), 1335.

8 A. P. Pêgo, A. A. Poot, D. W. Grijpma and J. Feijen, $J$. Controlled Release, 2003, 87(1-3), 69-79.

9 E. S. Place, J. H. George, C. K. Williams and M. M. Stevens, Chem. Soc. Rev., 2009, 38(4), 1139-1151.

10 K. Fukushima, Biomater. Sci., 2016, 4, 9-24.

11 J. Feng, R. X. Zhuo and X. Z. Zhang, Prog. Polym. Sci., 2012, 37(2), 211-236.

12 D. M. Stevens, S. Tempelaar, A. P. Dove and E. Harth, ACS Macro Lett., 2012, 1, 915-918.

13 V. X. Truong, I. A. Barker, M. Tan, L. Mespouille, P. Dubois and A. P. Dove, J. Mater. Chem. B, 2013, 1, 221-229.
14 D. M. Stevens, A. Rahalkar, B. Spears, K. Gilmore, E. Douglas, M. Muthukumar and E. Harth, Polym. Chem., 2015, 6, 10961102.

15 I. A. Barker, M. P. Ablett, H. T. J. Gilbert, S. J. Leigh, J. A. Covington, J. A. Hoyland, S. M. Richardson and A. P. Dove, Biomater. Sci., 2014, 2, 472-475.

16 H. Oie, A. Sudo and T. Endo, J. Polym. Sci., Part A: Polym. Chem., 2013, 51(9), 2035-2039.

17 K. Olofsson, M. Malkoch and A. J. Hult, J. Polym. Sci., Part A: Polym. Chem., 2016, 54(15), 2370-2378.

18 D. J. Darensbourg and Y. Wang, Polym. Chem., 2015, 6(10), 1768-1776.

19 C. Martín and A. W. Kleij, Macromolecules, 2016, 49(17), 6285-6295.

20 E. Bat, J. Feijen and D. W. Grijpma, Biomacromolecules, 2010, 11(10), 2692-2699. 